\title{
TRANSITIVE COURANT ALGEBROIDS
}

\author{
IZU VAISMAN
}

Received 28 September 2004 and in revised form 13 March 2005

We express any Courant algebroid bracket by means of a metric connection, and construct a Courant algebroid structure on any orthogonal, Whitney sum $E \oplus C$ where $E$ is a given Courant algebroid and $C$ is a flat, pseudo-Euclidean vector bundle. Then, we establish the general expression of the bracket of a transitive Courant algebroid, that is, a Courant algebroid with a surjective anchor, and describe a class of transitive Courant algebroids which are Whitney sums of a Courant subalgebroid with neutral metric and Courant-like bracket and a pseudo-Euclidean vector bundle with a flat, metric connection. In particular, this class contains all the transitive Courant algebroids of minimal rank; for these, the flat term mentioned above is zero. The results extend to regular Courant algebroids, that is, Courant algebroids with a constant rank anchor. The paper ends with miscellaneous remarks and an appendix on Dirac linear spaces.

\section{The basics of Courant algebroids}

The framework of this paper is the $C^{\infty}$-category. In the literature, there are two notions of a Courant algebroid, which include a skew-symmetric and a non-skew-symmetric bracket, respectively. These notions are the result of an effort to unify the Courant bracket and the Manin bracket [4].

We start with the definition of a non-skew-symmetric Courant algebroid $[6,7]$, with the simplifications indicated in [8].

Definition 1.1. A Courant algebroid is a pseudo-Euclidean vector bundle $(E \rightarrow M, g)$ ( $g$ is a symmetric, nondegenerate inner product on $E$ ) with an anchor morphism $\rho: E \rightarrow$ TM and a general, $\mathbb{R}$-bilinear product $\star: \Gamma E \times \Gamma E \rightarrow \Gamma E$ ( $\Gamma$ denotes spaces of global crosssections) such that for all $e, e_{1}, e_{2}, e_{3} \in \Gamma E$, the following properties hold:

(1) $(\rho e)\left(g\left(e_{1}, e_{2}\right)\right)=g\left(e \star e_{1}, e_{2}\right)+g\left(e_{1}, e \star e_{2}\right)$,

(2) $e \star e=\partial(g(e, e))$

(3) $e_{1} \star\left(e_{2} \star e_{3}\right)=\left(e_{1} \star e_{2}\right) \star e_{3}+e_{2} \star\left(e_{1} \star e_{3}\right)$, where $\partial=(1 / 2) \#_{g} \circ^{t} \rho: T^{*} M \rightarrow \Gamma E$ and

$$
\partial f=\partial(d f)=\frac{1}{2} \sharp_{g}{ }^{t} \rho(d f), \quad \forall f \in C^{\infty}(M) .
$$


Here, $t$ denotes transposition and $\#$ is the musical isomorphism defined like in Riemannian geometry.

By polarization, property (2) is equivalent with the prescription of the symmetric part of the product

$$
\left(e_{1}, e_{2}\right)_{*}=\frac{1}{2}\left(e_{1} \star e_{2}+e_{2} \star e_{1}\right)=\partial\left(g\left(e_{1}, e_{2}\right)\right)
$$

On the other hand, the definition of the operator $\partial$ is equivalent with

$$
g(\partial f, e)=\frac{1}{2}(\rho e) f, \quad \forall f \in C^{\infty}(M), \forall e \in \Gamma E .
$$

Hence, property (2) is also equivalent with

$$
g\left(e,\left(e_{1}, e_{2}\right)_{*}\right)=\frac{1}{2}(\rho e)\left(g\left(e_{1}, e_{2}\right)\right) .
$$

Thus, modulo property (1), we may replace (2) by a condition that does not contain $\partial$, namely,

$$
2 g\left(e,\left(e_{1}, e_{2}\right)_{*}\right)=g\left(e \star e_{1}, e_{2}\right)+g\left(e_{1}, e \star e_{2}\right) .
$$

We also consider the skew-symmetric part of the product

$$
\left[e_{1}, e_{2}\right]=\left[e_{1}, e_{2}\right]_{*}=\frac{1}{2}\left(e_{1} \star e_{2}-e_{2} \star e_{1}\right) .
$$

With (1.2) and (1.6), we get

$$
e_{1} \star e_{2}=\left[e_{1}, e_{2}\right]+\partial g\left(e_{1}, e_{2}\right) \text {. }
$$

From the properties postulated by Definition 1.1, one can deduce the following.

Proposition 1.2 [8]. Let $(E \rightarrow M, g, \rho, \star)$ be a Courant algebroid. Then, for all $e, e_{1}, e_{2} \in$ $\Gamma E$, for all $f \in C^{\infty}(M)$, the following properties hold:

(a) $e_{1} \star\left(f e_{2}\right)=f\left(e_{1} \star e_{2}\right)+\left(\left(\rho e_{1}\right) f\right) e_{2}$,

(b) $\left(f e_{1}\right) \star e_{2}=f\left(e_{1} \star e_{2}\right)-\left(\left(\rho e_{2}\right) f\right) e_{1}+2 g\left(e_{1}, e_{2}\right) \partial f$,

(c) $(\partial f) \star e=0, e \star(\partial f)=\partial((\rho e) f)$,

(d) $\rho\left(e_{1} \star e_{2}\right)=\left[\rho e_{1}, \rho e_{2}\right]_{\mathrm{TM}}$,

(e) $\rho(\partial f)=0$.

Proof. Property (a) follows from the comparison of the results of expressing $(\rho e)\left(g\left(f e_{1}\right.\right.$, $\left.e_{2}\right)$ ) in two ways, first by applying property (1) of Definition 1.1 straightforwardly, second by using the Leibniz rule for the vector field $\rho e$ applied to the product $f g\left(e_{1}, e_{2}\right)$ and then property (1) for $g\left(e_{1}, e_{2}\right)$.

Property (b) follows from (a) by using (1.2) and the fact that, on functions, $\partial$ satisfies the Leibniz rule.

Notice that properties (a) and (b) show that a Courant algebroid product is an operator of the local type (i.e., $\left(e_{1} \star e_{2}\right)(x)$ depends only on the restrictions of $e_{1}, e_{2}$ to a neighborhood of the point $x \in M)$. 
Now, we denote

$$
\mathscr{L}\left(e_{1}, e_{2}, e_{3}\right)=e_{1} \star\left(e_{2} \star e_{3}\right)-\left(e_{1} \star e_{2}\right) \star e_{3}-e_{2} \star\left(e_{1} \star e_{3}\right) .
$$

From (1.2), it follows that

$$
\mathscr{L}\left(e_{1}, e_{2}, e\right)+\mathscr{L}\left(e_{2}, e_{1}, e\right)=-2\left[\partial\left(g\left(e_{1}, e_{2}\right)\right) \star e\right] .
$$

Since any function $f \in C^{\infty}(M)$ may locally be written as

$$
f=g\left(e_{1}, \frac{f}{g\left(e_{1}, e_{1}\right)} e_{1}\right),
$$

where $e_{1}$ is not $g$-isotropic, (1.9) and property (3) of Definition 1.1 imply the first part of (c). The second part of (c) follows from the first part and relation (1.2).

In order to get property $(\mathrm{d})$ (where the right-hand side is a Lie bracket of vector fields on $M)$, we start with (1.3), written as

$$
\left(\rho e_{2}\right) f=2 g\left(\partial f, e_{2}\right)
$$

and apply $\rho e_{1}$, while using Definition 1.1(1) and (1.3) again. The result is

$$
\left(\rho e_{1}\right)\left(\rho e_{2}\right) f=2 g\left(e_{1} \star(\partial f), e_{2}\right)+\rho\left(e_{1} \star e_{2}\right) f .
$$

In view of the second part of (c), this relation becomes

$$
\left(\rho e_{1}\right)\left(\rho e_{2}\right) f=\rho\left(e_{1} \star e_{2}\right) f+\left(\rho e_{2}\right)\left(\rho e_{1}\right) f .
$$

Since $f$ is an arbitrary function, we got precisely (d).

Finally, from (b), we get

$$
\rho\left(\left(f e_{1}\right) \star e_{2}\right)=f \rho\left(e_{1} \star e_{2}\right)-\left(\left(\rho e_{2}\right) f\right) \rho e_{1}+2 g\left(e_{1}, e_{2}\right) \rho(\partial f)
$$

and, if we use (d) in the two sides of the previous relation while assuming $g\left(e_{1}, e_{2}\right) \neq 0$, we deduce property (e).

Remark 1.3. Property (c) implies that the skew-symmetric part of a Courant algebroid product satisfies the property

$$
[e, \partial f]=\frac{1}{2} \partial((\rho e) f) \text {. }
$$

Property (e) is equivalent with $g(\partial f, \partial g)=0$, for all $f, g \in C^{\infty}(M)$, that is, with the fact that im $\partial_{x}$ is a $g$-isotropic subspace of the fiber $E_{x}$, for all $x \in M$. We also note that property (e) is implied by (b) and (d). Finally, the computation used in the proof of (d) is reversible in the sense that (d) and formula (1.12) imply property (c) as well as its consequence (1.15). 
Remark 1.4. If $\rho=0$, we have $\partial=0$ and the Courant algebroid is just a bundle of Lie algebras with a pseudo-Euclidean metric $g$ that is invariant for the Lie algebra structure of each fiber.

From Definition 1.1 and Proposition 1.2, it follows that the skew-symmetric part (1.6) of a product $\star$ has the properties indicated by the following proposition.

Proposition 1.5 [6]. For any Courant algebroid and for all $e, e_{1}, e_{2}, e_{3} \in \Gamma E$, for all $f \in$ $C^{\infty}(M)$, the following properties hold:

(i) $\rho\left[e_{1}, e_{2}\right]=\left[\rho e_{1}, \rho e_{2}\right]$,

(ii) $\operatorname{im}\left(\#_{g} \circ f\right) \subseteq \operatorname{ker} \rho$,

(iii) $\sum_{\text {Cycl }}\left[\left[e_{1}, e_{2}\right], e_{3}\right]=(1 / 3) \partial\left[\sum_{\mathrm{Cycl}} g\left(\left[e_{1}, e_{2}\right], e_{3}\right)\right]$,

(iv) $\left[e_{1}, f e_{2}\right]=f\left[e_{1}, e_{2}\right]+\left(\left(\rho e_{1}\right) f\right) e_{2}-g\left(e_{1}, e_{2}\right) \partial f$,

(v) $(\rho e)\left[g\left(e_{1}, e_{2}\right)\right]=g\left(\left[e, e_{1}\right]+\partial g\left(e, e_{1}\right), e_{2}\right)+g\left(e_{1},\left[e, e_{2}\right]+\partial g\left(e, e_{2}\right)\right)$.

Proof. Except for (iii), these properties are immediate consequences of Definition 1.1 and Proposition 1.2. For (iii), we denote

$$
\mathscr{F}\left(e_{1}, e_{2}, e_{3}\right)=\left[\left[e_{1}, e_{2}\right], e_{3}\right]+\left[\left[e_{2}, e_{3}\right], e_{1}\right]+\left[\left[e_{3}, e_{1}\right], e_{2}\right] .
$$

Using (1.7), we get

$$
\begin{aligned}
\mathscr{L}\left(e_{1}, e_{2}, e_{3}\right)= & \sum_{\operatorname{Cycl}(1,2,-3)} \partial\left(g\left(e_{1},\left[e_{2}, e_{3}\right]\right)\right)+\sum_{\operatorname{Cycl}(1,-2,3)}\left[e_{1}, \partial\left(g\left(e_{2}, e_{3}\right)\right)\right] \\
& -\frac{1}{2} \sum_{\operatorname{Cycl}(-1,2,3)} \partial\left(\left(\rho e_{1}\right)\left(g\left(e_{2}, e_{3}\right)\right)\right)-\mathscr{J}\left(e_{1}, e_{2}, e_{3}\right),
\end{aligned}
$$

where, in the sums of the right-hand side, the indices $(1,2,3)$ move cyclically while the signs of the terms are as indicated in the summation index.

Now, taking the sum of (1.17) over cyclic permutations of $(1,2,3)$ and using (1.9), we get

$$
6 \mathscr{F}\left(e_{1}, e_{2}, e_{3}\right)=\sum_{\operatorname{Cycl}(1,2,3)}\left\{\mathscr{L}\left(e_{1}, e_{2}, e_{3}\right)-\mathscr{L}\left(e_{2}, e_{1}, e_{3}\right)+2 \partial\left(g\left(e_{1},\left[e_{2}, e_{3}\right]\right)\right)\right\} .
$$

Formula (1.18) shows that property (3) of Definition 1.1 implies the present property (iii).

Definition 1.6 [4]. A skew-symmetric Courant algebroid is a pseudo-Euclidean vector bundle $(E \rightarrow M, g)$ with an anchor morphism $\rho: E \rightarrow$ TM and a skew-symmetric bracket $[\cdot, \cdot \cdot]$ on $\Gamma E$ such that properties (i)-(v) of Proposition 1.5 hold. 
Remark 1.7 [8]. With the same proofs as for Proposition 1.2, we can see that property (v) of Proposition 1.5 implies (iv) and properties (i) and (iv) imply (ii). Therefore, conditions (i), (iii), and (v) suffice in the definition of a skew-symmetric Courant algebroid.

Remark 1.8. Just like in the case of a Lie algebroid, for a skew-symmetric Courant algebroid, $\mathscr{D}=\operatorname{im} \rho$ is a generalized foliation on $M$. Indeed, from property (i), Proposition 1.5, it follows that $\mathscr{D}=\operatorname{span}\{\rho e / \forall e \in \Gamma E\}$ is spanned by a Lie algebra, and, for any vector field $X=\rho e$, for all $x \in M$, $\operatorname{dim} \mathscr{D}_{(\exp (t X))(x)}=\left.\operatorname{rank} \rho\right|_{(\exp (t X))(x)}=$ const. (For the latter assertion, look at a Lie derivative of $\rho$ defined as for a tensor field and notice that this Lie derivative vanishes.) Then the result follows from the Sussmann-Stefan-Frobenius theorem (e.g., [10, Theorem 2.9"]).

For simplicity, in what follows, we will use the language provided by the next definitions.

Definition 1.9. A Courant anchor is a vector bundle morphism $\rho: E \rightarrow \mathrm{TM}$, where $(E, g)$ is a pseudo-Euclidean vector bundle over the manifold $M$, which is such that $\operatorname{im} \rho$ is a (generalized) foliation and the corresponding morphism $\partial: T^{*} M \rightarrow E$ defined by (1.1) has a $g$-isotropic image. A triple $(E, g, \rho)$ where $\rho$ is a Courant anchor will be called a Courant vector bundle. A Courant vector bundle endowed with a skew-symmetric bracket $[\cdot, \cdot]$ on $\Gamma E$, which satisfies properties (v) and (i) (therefore, also, (iv) and (ii)) of a skewsymmetric Courant algebroid will be called a pre-Courant algebroid.

Notice that, since, for all $x \in M$, both $\operatorname{im} \rho$ and im $\partial$ have the dimension equal to rank $\rho$, a Courant vector bundle must satisfy the condition $\operatorname{rank} \rho \leq b \leq(1 / 2) \operatorname{rank} E$, where $b$ is the smallest between the positive-negative inertia indices of $g$. Furthermore, in view of Remark 1.3, formula (1.15) holds for any pre-Courant algebroid. Using (1.15) it is easy to check that any pre-Courant algebroid satisfies property (iii) of Proposition 1.5 if at least one of the arguments $e_{a} \in \operatorname{im} \partial(a=1,2,3)$ (the cross-sections of the subbundle im $\partial$ are locally spanned over $C^{\infty}(M)$ by cross-sections of the form $\left.\partial f\left(f \in C^{\infty}(M)\right)\right)$.

By Proposition 1.5, the skew-symmetric part of a Courant algebroid product yields a skew-symmetric Courant algebroid bracket. The converse is also true.

Proposition 1.10 (e.g., [6]). If the bracket [·, · ] satisfies the properties of a skew-symmetric Courant algebroid, the product $\star$ defined by (1.7) satisfies the properties of a Courant algebroid.

Proof. Obviously, Definition 1.1(1) and (2) hold. Moreover, as explained in Remark 1.3, property (c) of Proposition 1.2 holds independently of formula (1.9). Accordingly, now, (1.9) proves that

$$
\mathscr{L}\left(e_{1}, e_{2}, e_{3}\right)+\mathscr{L}\left(e_{2}, e_{1}, e_{3}\right)=0 .
$$

On the other hand, if Proposition 1.5(iii) holds, formula (1.18) implies

$$
\mathscr{L}\left(e_{1}, e_{2}, e_{3}\right)-\mathscr{L}\left(e_{2}, e_{1}, e_{3}\right)=0
$$

Hence, we are done. 
The following proposition shows the possible changes of the bracket of a pre-Courant algebroid.

Proposition 1.11. Let $(E, g, \rho,[\cdot, \cdot])$ be a pre-Courant algebroid. Then, the formula

$$
\left[e_{1}, e_{2}\right]^{\prime}=\left[e_{1}, e_{2}\right]+\lambda\left(e_{1}, e_{2}\right), \quad e_{1}, e_{2} \in \Gamma E,
$$

where $\lambda \in \Gamma\left(\wedge^{2} E^{*} \otimes E\right), \rho \circ \lambda=0$, and

$$
\Lambda\left(e_{1}, e_{2}, e_{3}\right)=g\left(\lambda\left(e_{1}, e_{2}\right), e_{3}\right)
$$

is totally skew-symmetric (i.e., $\Lambda \in \Gamma \wedge^{3} E^{*}$ ), yields all the pre-Courant algebroid structures on $(E, g, \rho)$. Furthermore, the bracket (1.21) is that of a Courant algebroid if and only if

$$
\left(\partial_{[]} \Lambda\right)\left(e_{1}, e_{2}, e_{3}\right)=\mathscr{g}\left(e_{1}, e_{2}, e_{3}\right)-\frac{1}{3} \partial\left[\sum_{\text {Cycl }} g\left(\left[e_{1}, e_{2}\right], e_{3}\right)\right],
$$

where

$$
\begin{aligned}
\left(\partial_{[]} \Lambda\right)\left(e_{1}, e_{2}, e_{3}\right)=\partial\left(\Lambda\left(e_{1}, e_{2}, e_{3}\right)\right) & \\
-\sum_{\operatorname{Cycl}(1,2,3)}\{ & \lambda\left(\lambda\left(e_{1}, e_{2}\right), e_{3}\right)+\lambda\left(\left[e_{1}, e_{2}\right], e_{3}\right) \\
+ & {\left.\left[\lambda\left(e_{1}, e_{2}\right), e_{3}\right]\right\} \in \Gamma\left(\wedge^{3} E^{*} \otimes E\right) . }
\end{aligned}
$$

Proof. The difference of two brackets of pre-Courant algebroid structures is a form $\lambda \in$ $\Gamma\left(\wedge^{2} E^{*} \otimes E\right)$ because of property (iv), Proposition 1.5. The indicated conditions for $\lambda$ are equivalent with properties (i) and (v) of the same proposition, respectively. Notice that $\rho \circ \lambda=0$ is equivalent with

$$
\Lambda\left(e_{1}, e_{2}, \partial f\right)=0, \quad \forall f \in C^{\infty}(M),
$$

therefore, in view of the skew-symmetry of $\Lambda$, is also equivalent with

$$
\lambda(e, \partial f)=0, \quad \forall e \in \Gamma E, \forall f \in C^{\infty}(M) .
$$

Finally, a technical computation shows that property (iii) is equivalent with (1.23) and (1.24).

Remark $1.12[1,4]$. Any $g$-isotropic subalgebroid (i.e., a vector subbundle that is closed by brackets) of a skew-symmetric Courant algebroid is a Lie algebroid. 


\section{Courant brackets and metric connections}

We will get further insight into the structure of the bracket of a Courant algebroid by using a metric connection $\nabla$ on the pseudo-Euclidean bundle $(E, g)$, which means that

$$
X\left(g\left(e_{1}, e_{2}\right)\right)=g\left(\nabla_{X} e_{1}, e_{2}\right)+g\left(e_{1}, \nabla_{X} e_{2}\right) \quad(X \in \Gamma \mathrm{TM}) .
$$

If we also have the morphism $\rho: E \rightarrow \mathrm{TM}$, we define the $\rho$-torsion $T_{(\nabla, \rho)} \in \Gamma\left(\wedge^{2} E^{*} \otimes \mathrm{TM}\right)$ by the formula

$$
T_{(\nabla, \rho)}\left(e_{1}, e_{2}\right)=\rho\left(\nabla_{\rho e_{1}} e_{2}-\nabla_{\rho e_{2}} e_{1}\right)-\left[\rho e_{1}, \rho e_{2}\right] .
$$

Now, we can prove the following.

Proposition 2.1. (1) The formula

$$
\left[e_{1}, e_{2}\right]_{0}=\nabla_{\rho e_{1}} e_{2}-\nabla_{\rho e_{2}} e_{1}-\gamma\left(e_{1}, e_{2}\right)
$$

where $\gamma$ is defined by the equality

$$
g\left(\gamma\left(e_{1}, e_{2}\right), e\right)=\frac{1}{2}\left[g\left(e_{1}, \nabla_{\rho e} e_{2}\right)-g\left(e_{2}, \nabla_{\rho e} e_{1}\right)\right], \quad \forall e \in \Gamma E,
$$

defines a skew-symmetric bracket on $\Gamma E$ that satisfies property $(v)$ of Proposition 1.5.

(2) The most general bracket that satisfies ( $v)$ is given by

$$
\left[e_{1}, e_{2}\right]=\left[e_{1}, e_{2}\right]_{0}-\beta\left(e_{1}, e_{2}\right),
$$

where $\beta \in \Gamma\left(\wedge^{2} E^{*} \otimes E\right)$ and

$$
B\left(e_{1}, e_{2}, e_{3}\right)=g\left(\beta\left(e_{1}, e_{2}\right), e_{3}\right)
$$

is totally skew-symmetric, that is, $B \in \Gamma \wedge^{3} E^{*}$.

(3) The bracket (2.5) also satisfies property (i) of Proposition 1.5 if and only if the following two equalities hold:

$$
\begin{gathered}
\rho \circ \partial=0, \\
\rho\left(\beta\left(e_{1}, e_{2}\right)\right)=T_{(\nabla, \rho)}\left(e_{1}, e_{2}\right), \quad \forall e_{1}, e_{2} \in \Gamma E,
\end{gathered}
$$

where $T_{(\nabla, \rho)}$ is the $\rho$-torsion of $\nabla$. In particular, if $(E, g, \rho)$ has a metric connection $\nabla$ with zero $\rho$-torsion, the bracket (2.3) satisfies property (i).

Proof. First, we notice that formula (2.4) actually defines $\gamma$ because if this formula holds for $e \in \Gamma E$, it also holds for $f e$, for all $f \in C^{\infty}(M)$, and since $g$ is nondegenerate. Then, a technical calculation, which takes into account the metric property (2.1), shows that the bracket (2.3) satisfies property $(\mathrm{v})$.

Now, the difference $\beta$ between two brackets that satisfy (v) is such that the corresponding $B$ given by (2.6) is totally skew-symmetric, and $\beta \in \Gamma\left(\wedge^{2} E^{*} \otimes E\right)$, respectively, $B \in \wedge^{3} E^{*}$, because (v) implies property (iv) of Proposition 1.5. These remarks justify the general formula (2.5). 
Finally, from (2.5), we get

$$
\rho\left[e_{1}, e_{2}\right]=\left[\rho e_{1}, \rho e_{2}\right]+T_{\nabla, \rho}\left(e_{1}, e_{2}\right)-\rho\left(\gamma\left(e_{1}, e_{2}\right)\right)-\rho\left(\beta\left(e_{1}, e_{2}\right)\right) .
$$

We know that if properties (i) and (v) of Proposition 1.5 hold, so does (iv) and then (ii), and (ii) is equivalent with (2.7). Then, by using (2.4) for $e=\partial f, f \in C^{\infty}(M)$, we get $\rho \circ \gamma=0$, and (2.9) implies (2.8). The converse also is clear from (2.9). The last assertion follows since, if $T_{(\nabla, \rho)}=0$, we may chose $\beta=0$.

Remark 2.2. By applying the vector fields of formula (2.8) to an arbitrary function $f$, it follows that (2.8) is equivalent with

$$
B\left(e_{1}, e_{2}, \partial f\right)=\frac{1}{2}\left(T_{(\nabla, \rho)}\left(e_{1}, e_{2}\right)\right) f, \quad e_{1}, e_{2} \in \Gamma E, f \in C^{\infty}(M) .
$$

Formula (2.5), where $B$ defined by (2.6) is skew-symmetric and (2.7), (2.8) hold, defines all the pre-Courant algebroid brackets on a given Courant vector bundle. Among them, the Courant algebroid brackets are obtained if condition (iii) of Proposition 1.5 also holds. In order to express the latter, we denote

$$
\mathscr{C}\left(e_{1}, e_{2}, e_{3}\right)=\mathscr{J}\left(e_{1}, e_{2}, e_{3}\right)-\frac{1}{3} \partial\left[\sum_{\operatorname{Cycl}(1,2,3)} g\left(\left[e_{1}, e_{2}\right], e_{3}\right)\right],
$$

where $\mathscr{F}$ is defined by (1.16), and denote by an index 0 the same expression for the bracket $[\cdot, \cdot]_{0}$. Assuming that property (i) holds, it follows that

$$
\mathscr{C}_{0}\left(e_{1}, e_{2}, e_{3}\right)=\sum_{\operatorname{Cycl}(1,2,3)}\left\{\left(\nabla_{\rho e_{3}} \gamma\right)\left(e_{1}, e_{2}\right)+\gamma\left(\gamma\left(e_{1}, e_{2}\right), e_{3}\right)-R_{\nabla}\left(\rho e_{1}, \rho e_{2}\right) e_{3}\right\}
$$

where $R_{\nabla}$ is the curvature of the connection $\nabla$ and $\nabla \gamma$ is defined as if $\gamma$ would be a tensor. In order to check formula (2.12), we express $g\left(\mathscr{C}_{0}\left(e_{1}, e_{2}, e_{3}\right), e\right)$ for an arbitrary vector $e$, and use (1.3) and the metric character of $\nabla$. The computation is lengthy but technical. Furthermore, if the brackets of (2.11) are replaced by their expression (2.5), and the terms of the resulting formula are grouped conveniently, one gets

$$
\begin{aligned}
\mathscr{C}\left(e_{1}, e_{2}, e_{3}\right)= & \mathscr{C}_{0}\left(e_{1}, e_{2}, e_{3}\right) \\
& +\sum_{\operatorname{Cycl}(1,2,3)}\left\{\beta\left(\beta\left(e_{1}, e_{2}\right), e_{3}\right)-\beta\left(\left[e_{1}, e_{2}\right]_{0}, e_{3}\right)-\left[\beta\left(e_{1}, e_{2}\right), e_{3}\right]_{0}\right\} .
\end{aligned}
$$

These formulas express the condition for the bracket (2.5) to satisfy property (iii), $\mathscr{b}=0$, by means of the metric connection $\nabla$. In particular, if there exists a metric connection of zero $\rho$-torsion, the corresponding bracket (2.3), which satisfies (i), also satisfies (iii) if and only if $\mathscr{C}_{0}=0$, and this condition reminds us of the Bianchi identity for a linear connection with torsion on a differentiable manifold $M$. 
As an application of the results given in this section, we have the following.

Proposition 2.3. Let $(E, g, \rho,[\cdot, \cdot])$ be a pre-Courant algebroid. Then, for any pseudoEuclidean vector bundle $\left(C, g_{0}\right)$ over $M$ and any metric connection $\nabla$ on $C$, the brackets

$$
\left[e_{1}, e_{2}\right],[e, c]=-[c, e]=\nabla_{\rho e} \mathcal{c}, \quad\left[c_{1}, c_{2}\right]=-\gamma_{0}\left(c_{1}, c_{2}\right)
$$

where $e, e_{1}, e_{2} \in \Gamma E, c, c_{1}, c_{2} \in \Gamma C$, and $\gamma_{0}\left(c_{1}, c_{2}\right) \in \Gamma E$ is defined by

$$
g\left(\gamma_{0}\left(c_{1}, c_{2}\right), e\right)=\frac{1}{2}\left[g_{0}\left(c_{1}, \nabla_{\rho e} c_{2}\right)-g_{0}\left(c_{2}, \nabla_{\rho e} c_{1}\right)\right]
$$

define a pre-Courant algebroid structure on $\left(E \oplus C, g \oplus g_{0}, \rho \oplus 0\right)$. If the original algebroid is a Courant algebroid, (2.14) yields a Courant algebroid structure if and only if the connection $\nabla$ is flat.

Proof. Straightforward checks show that the brackets (2.14) satisfy property (v) of a preCourant algebroid. For a full justification of property (i), we must also notice that $\rho\left(\gamma_{0}\left(c_{1}\right.\right.$, $\left.\left.c_{2}\right)\right)=0$. This follows since (2.15) implies $g\left(\gamma_{0}\left(c_{1}, c_{2}\right), \partial f\right)=0$, for all $f \in C^{\infty}(M)$. For the last assertion of the proposition, we refer to property (iii) of a Courant algebroid. For arguments $e_{1}, e_{2}, e_{3}$, (iii) holds if $E$ is a Courant algebroid and for arguments $c_{1}, c_{2}, c_{3}$, (iii) follows from $\rho\left(\gamma_{0}\left(c_{1}, c_{2}\right)\right)=0$. For arguments $e_{1}, e_{2}, c$, (iii) is just $R_{\nabla}\left(\rho e_{1}, \rho e_{2}\right) c=0$, where $R_{\nabla}$ is the curvature of $\nabla$. Finally, for arguments $e, c_{1}, c_{2}$, (iii) means

$$
\left[e, \gamma_{0}\left(c_{1}, c_{2}\right)\right]-\gamma_{0}\left(\nabla_{\rho e} c_{1}, c_{2}\right)-\gamma_{0}\left(c_{1}, \nabla_{\rho e} c_{2}\right)-\frac{1}{2} \partial\left\{g_{0}\left(\nabla_{\rho e} c_{1}, c_{2}\right)-g_{0}\left(c_{1}, \nabla_{\rho e} c_{2}\right)\right\}=0 .
$$

Since for a pre-Courant algebroid property (iv) holds, the left-hand side of the previous equality is $C^{\infty}(M)$-linear and it suffices to check it for a local basis of $C$. If connection $\nabla$ is flat, $C$ has local $\nabla$-parallel bases and (2.16) obviously holds.

\section{Transitive and regular Courant algebroids}

In this section, we determine the structure of the transitive Courant algebroids, that is, Courant algebroids with a surjective anchor. The results may then be extended to regular Courant algebroids, that is, Courant algebroids with a constant rank anchor. When this paper was ready, I was informed that the transitive Courant algebroids were also determined by Ševera in unpublished correspondence with Weinstein, without metric connections (see Remark 3.6 later on).

Let $(E, g, \rho)$ be a Courant vector bundle with a surjective anchor $\rho$. Then, $K=\operatorname{ker} \rho$ is a (regular) subbundle of $E$ of $\operatorname{rank} k=r-n$, where $r=\operatorname{rank} E$ and $n=\operatorname{dim} M$, and, if $K^{\perp_{g}}$ is the $g$-orthogonal subbundle of $K, \operatorname{rank}\left(K \cap K^{\perp_{g}}\right)=r-s$, where $s=\operatorname{rank}\left(K+K^{\perp_{g}}\right)$. In view of the properties of a Courant anchor $\operatorname{rank}(\operatorname{im} \partial)=n$ and $\operatorname{im} \partial \subseteq\left(K \cap K^{\perp_{g}}\right)$, whence it follows easily that $s=k$. Accordingly, $K$ is a $g$-coisotropic subbundle of $E$ and $K^{\perp_{g}}=$ $\operatorname{im} \partial \subseteq K$. 
In this situation, it is known that there exists an isotropic, complementary subbundle $Q$ of $K$ in $E$ and a complementary subbundle $C$ of im $\partial$ in $K$ such that

$$
E=P \oplus C \quad(P=\operatorname{im} \partial \oplus Q)
$$

is a $g$-orthogonal decomposition, the restriction of $g$ to $C$ is nondegenerate, and the restriction of $g$ to $P$ is nondegenerate and neutral (i.e., of signature zero). We will say that $P$ is a neutral completion of im $\partial$. (The reader may see [9] for similar results in the symplectic case and the proofs are the same in the pseudo-Euclidean case.) For what follows, we fix a decomposition (3.1), which means that we also have $E=K \oplus Q$, and $\left.\rho\right|_{Q}$ is an isomorphism with the inverse $\sigma: \mathrm{TM} \rightarrow Q$. We will denote by $p_{K}, p_{Q}, p_{P}, p_{\text {im } \partial,} p_{C}$ the projections of $E$ onto the corresponding subspaces, respectively.

Furthermore, we construct a metric connection $\nabla$ of $E$ as follows. $\nabla$ will be the sum of metric connections of the components $P, C$. Furthermore, the component $\nabla^{P}$ will be a sum $\nabla^{\operatorname{im} \partial} \oplus \nabla^{Q}$ where $\nabla^{Q}$ is arbitrary and $\nabla^{\mathrm{im} \partial}$ is defined by the condition

$$
X(g(q, \partial f))=g\left(q, \nabla_{X}^{\operatorname{im} \partial}(\partial f)\right)+g\left(\nabla_{X}^{Q} q, \partial f\right) \quad\left(q \in \Gamma Q, X \in \Gamma \mathrm{TM}, f \in C^{\infty}(M)\right) .
$$

This condition defines well $\nabla^{\text {im } \partial}$ because $\left.g\right|_{P}$ is neutral. A metric connection on $(E, g)$ which is obtained by the process described above is said to be suitable, and we fix one such suitable connection.

The component $\nabla^{Q}$ of a suitable connection may be identified with a linear connection $D$ on $M$ by means of the formula

$$
D_{X} Y=\rho\left(\nabla_{X}(\sigma Y)\right) \quad(X, Y \in \Gamma \mathrm{TM}) .
$$

The following formula defines a 3-form $B_{1} \in \Gamma \wedge^{3} E^{*}$ :

$$
B_{1}\left(e_{1}, e_{2}, e_{3}\right)=\sum_{\operatorname{Cycl}(1,2,3)} g\left(\sigma\left(T_{(\nabla, \rho)}\left(e_{1}, e_{2}\right)\right), p_{\operatorname{im} \partial}\left(e_{3}\right)\right) .
$$

From the fact that the $\nabla$-parallel translations preserve the subbundle im $\partial$, and formula (2.2), it follows that $B_{1}$ satisfies condition (2.10). Hence, formula (2.5) with $\beta=\beta_{1}$ defined by $B_{1}$ yields a structure of a pre-Courant algebroid on $(E, g, \rho)$, with a bracket that we denote by $[\cdot, \cdot]_{1}$.

Like for any pre-Courant algebroid, in the transitive case too, the brackets $[e, \partial f]$ are always given by formula (1.15). Furthermore, we get the following.

Proposition 3.1. The bracket $[\cdot, \cdot]_{1}$ is defined by the formulas

$$
\begin{aligned}
p_{Q}\left[e_{1}, e_{2}\right]_{1}= & p_{Q} \nabla_{\rho e_{1}} e_{2}-p_{Q} \nabla_{\rho e_{2}} e_{1}-\sigma\left(T_{(\nabla, \rho)}\left(e_{1}, e_{2}\right)\right), \quad \forall e_{1}, e_{2} \in \Gamma E, \\
g\left(p_{K}\left[e_{1}, e_{2}\right]_{1}, e\right)= & g\left(\nabla_{\rho e_{1}} e_{2}, e\right)-g\left(\nabla_{\rho e_{2}} e_{1}, e\right)-\frac{1}{2} g\left(\nabla_{\rho e} e_{2}, e_{1}\right)+\frac{1}{2} g\left(\nabla_{\rho e} e_{1}, e_{2}\right) \\
& -g\left(p_{Q} \nabla_{\rho e_{1}} e_{2}, e\right)+g\left(p_{Q} \nabla_{\rho e_{2}} e_{1}, e\right)+g\left(\sigma T_{(\nabla, \rho)}\left(e_{1}, e\right), e_{2}\right) \\
& -g\left(\sigma T_{(\nabla, \rho)}\left(e_{2}, e\right), e_{1}\right),
\end{aligned}
$$

where $e \in \Gamma E$ and we use an arbitrary decomposition (3.1) and an arbitrary suitable connection $\nabla$. 
Proof. The first formula follows by applying $\sigma$ to (2.2), since $\sigma \circ \rho$ is the projection $p_{Q}$ and the bracket $[\cdot, \cdot]$ satisfies property $(\mathrm{i})$.

Furthermore, if we expend the expression of the bracket $[\cdot, \cdot]_{1}$, take the scalar $g$ product by an arbitrary $e \in \Gamma E$, and use formula (3.5), we see that (3.6) also holds.

Formulas (3.5) and (3.6) define the projections of the bracket $[\cdot, \cdot]_{1}$ on $Q$ and $K$, hence completely define the bracket.

In the following proposition, we give a more transparent expression of the bracket $[\cdot, \cdot]_{1}$.

Proposition 3.2. Let $(E, g, \rho)$ be a Courant vector bundle with a surjective anchor, for which a choice of a splitting (3.1) and of a suitable connection $\nabla$ is made. Then, $(E, g, \rho)$ has a structure of pre-Courant algebroid with the bracket $[\cdot, \cdot]_{1}$ given by the formulas

$$
\begin{aligned}
{\left[q_{1}+\partial f_{1}, q_{2}+\partial f_{2}\right]_{1} } & =\sigma\left[\rho q_{1}, \rho q_{2}\right]+\frac{1}{2}\left[\partial\left(\left(\rho q_{1}\right) f_{2}\right)-\partial\left(\left(\rho q_{2}\right) f_{1}\right)\right], \\
{\left[c_{1}, c_{2}\right]_{1} } & =-\gamma\left(c_{1}, c_{2}\right), \quad[c, q+\partial f]_{1}=-\nabla_{\rho q} c
\end{aligned}
$$

where $f, f_{1}, f_{2} \in C^{\infty}(M) ; q, q_{1}, q_{2} \in \Gamma Q ; c, c_{1}, c_{2} \in \Gamma C$, and $\gamma$ is defined by (2.4). Furthermore, $[\cdot, \cdot]_{1}$ is a Courant algebroid bracket if and only if the $C$-component of the connection $\nabla$ is flat.

Proof. In view of the properties of decomposition (3.1), $k \in K$ is completely defined by the scalar products $g(k, q)$ for all $q \in Q$. But, if $e, e_{1}, e_{2}$ of (3.6) are in $Q$, using the definition of a suitable connection, we get $p_{K}\left[q_{1}, q_{2}\right]_{1}=0$.

Accordingly, with (3.5) and (2.2), we get $\left[q_{1}, q_{2}\right]_{1}=\sigma\left[\rho q_{1}, \rho q_{2}\right]$ and the first formula (3.7) follows if we also take into account (1.15).

By similar considerations based on the properties of decomposition (3.1) and of a suitable connection, (3.5), (3.6), and (1.15) yield the remaining formulas (3.7).

Of course, brackets $[\cdot, \cdot]_{1}$ with general factors $\sum_{i} h_{i} \partial f_{i} \in \operatorname{im} \partial$ will be deduced from (3.7) by means of property (iv), Proposition 1.5. (Alternatively, we may use again (3.5) and (3.6).) Notice also that (2.4) implies $\gamma\left(e_{1}, e_{2}\right) \in K^{\perp_{g}}=\operatorname{im} \partial$, for all $e_{1}, e_{2} \in \Gamma E$.

From the first formula (3.7), we see that $\left(P,\left.g\right|_{P},\left.\rho\right|_{P}\right)(P=Q \oplus$ im $\partial)$ is a pre-Courant algebroid with the induced bracket. Moreover, it is easy to check that property (iii) of Proposition 1.5 also holds on $P$, therefore we actually have a Courant algebroid $P$, and (3.7) is the structure defined on $P \oplus Q$ by Proposition 2.3. Accordingly, the last assertion of the present proposition follows from the last assertion of Proposition 2.3.

As shown by Proposition 1.11, all the other pre-Courant brackets of the Courant bundle with surjective anchor $(E, g, \rho)$ will be obtained from $(3.7)$ by the addition of a form $\lambda$ that satisfies the corresponding hypotheses, whence $\lambda \in \Gamma\left(\wedge^{2} E^{*} \otimes K\right)$ and (1.26) holds. The corresponding formulas are

$$
\begin{aligned}
{\left[q_{1}+\partial f_{1}, q_{2}+\partial f_{2}\right] } & =\sigma\left[\rho q_{1}, \rho q_{2}\right]+\lambda\left(q_{1}, q_{2}\right)+\frac{1}{2}\left[\partial\left(\left(\rho q_{1}\right) f_{2}\right)-\partial\left(\left(\rho q_{2}\right) f_{1}\right)\right], \\
{\left[c_{1}, c_{2}\right] } & =-\gamma\left(c_{1}, c_{2}\right)+\lambda\left(c_{1}, c_{2}\right), \quad[c, q+\partial f]=-\nabla_{\rho q} c+\lambda(c, q) .
\end{aligned}
$$


In order to get Courant algebroid brackets, we must ask $\lambda$ to satisfy condition (1.23). If expressed on arguments $q \in \Gamma Q, c \in \Gamma C$, (1.23) decomposes into the following four components:

$$
\begin{gathered}
\left(\partial_{[]} \Lambda\right)\left(q_{1}, q_{2}, q_{3}\right)=0 \\
\left(\partial_{[]} \Lambda\right)\left(q_{1}, q_{2}, c\right)=-R_{\nabla}\left(\rho q_{1}, \rho q_{2}\right) c \\
\left(\partial_{[]} \Lambda\right)\left(q, c_{1}, c_{2}\right)=\partial\left[g\left(q, \gamma\left(c_{1}, c_{2}\right)\right)\right]_{1}+\left[q, \gamma\left(c_{1}, c_{2}\right)\right]_{1}-\gamma\left(\nabla_{\rho q} c_{1}, c_{2}\right)-\gamma\left(c_{1}, \nabla_{\rho q} c_{2}\right), \\
\left(\partial_{[]} \Lambda\right)\left(c_{1}, c_{2}, c_{3}\right)=\sum_{\operatorname{Cycl}(1,2,3)}\left[c_{1}, \gamma\left(c_{2}, c_{3}\right)\right]_{1} .
\end{gathered}
$$

In principle, formulas (3.8) and (3.9)-(3.12) yield all the transitive Courant algebroids.

We get a more transparent result if we restrict ourselves to the subclass of transitive Courant algebroids that admit a bracket-closed neutral extension of the subbundle im $\partial$. We will call them transitive, restricted, Courant algebroids.

The general bracket (3.8) is restricted if and only if it is defined by a form $\lambda$ such that, for all $q_{1}, q_{2} \in \Gamma Q, \lambda\left(q_{1}, q_{2}\right) \in \operatorname{im} \partial$. This condition is equivalent with $\Lambda\left(q_{1}, q_{2}, c\right)=0$, which, because of the skew-symmetry, is equivalent with $\lambda(c, q) \in \Gamma C$. Then, we may change the $C$-component of the connection $\nabla$ by $\nabla_{X} C \mapsto \nabla_{X} C+\lambda(c, \sigma(X))$ and get for the same bracket a simplified expression (3.8) that looks as if we have used an additional form $\lambda$ such that $\lambda(c, q)=0$ and, accordingly, $\lambda\left(c_{1}, c_{2}\right) \in \Gamma C$. Then, conditions (3.9)(3.12) become simpler and we obtain the following.

Proposition 3.3. The bracket (3.8) defines a transitive, restricted, Courant algebroid if and only if the C-component of the connection $\nabla$ is flat and, in addition to the conditions for a pre-Courant algebroid, the form $\lambda$ also satisfies the conditions

$$
\begin{gathered}
\lambda(c, q)=0, \quad \lambda\left(q_{1}, q_{2}\right) \in \Gamma(\operatorname{im} \partial), \quad \lambda\left(c_{1}, c_{2}\right) \in \Gamma C \\
\partial\left(\Lambda\left(q_{1}, q_{2}, q_{3}\right)\right)=\sum_{\operatorname{Cycl}(1,2,3)}\left\{\lambda\left(\sigma\left[\rho q_{1}, \rho q_{2}\right], q_{3}\right)-\left[q_{3}, \lambda\left(q_{1}, q_{2}\right)\right]\right\}, \\
\partial\left(\Lambda\left(c_{1}, c_{2}, c_{3}\right)\right)=-\sum_{\operatorname{Cycl}(1,2,3)} \gamma\left(\lambda\left(c_{1}, c_{2}\right), c_{3}\right), \\
\left(\nabla_{\rho q} \lambda\right)\left(c_{1}, c_{2}\right)=0 .
\end{gathered}
$$

Proof. Conditions (3.13) transform (3.10) into the flatness of $\nabla,(3.9)$ into (3.14), (3.12) into (3.15), and (3.11) into (3.16).

Corollary 3.4. A Courant vector bundle with surjective anchor has a restricted Courant algebroid structure if and only if it is a Whitney sum (3.1) of a Courant subalgebroid with neutral metric and a flat pseudo-Euclidean bundle. 
Proof. For a Courant algebroid, the conditions stated by the corollary were proven in Proposition 3.3. The bracket of the subalgebroid is defined by the first formula (3.8). Conversely, if the conditions hold, a corresponding Courant algebroid bracket is defined by (3.8) with $\lambda=0$.

Remark 3.5. The minimal possible rank of a transitive Courant algebroid over an $n$ dimensional manifold $M$ is $2 n$. Proposition 3.3 yields all the transitive Courant algebroids of minimal rank. Namely, they have no $C$-component and the bracket is defined by the first formula (3.8) where $\lambda\left(q_{1}, q_{2}\right) \in \operatorname{im} \partial$ satisfies condition (3.14).

Remark 3.6. With the notation used above, let $E$ be a transitive Courant algebroid. Then $E /$ im $\partial$ gets an induced structure of a transitive Lie algebroid and we have the exact sequence

$$
0 \longrightarrow \operatorname{im} \partial \stackrel{\subseteq}{\longrightarrow} E \stackrel{\pi}{\longrightarrow} E / \operatorname{im} \partial \longrightarrow 0 .
$$

Hence, $E$ may be seen as a central extension of a transitive Lie algebroid. In the restricted case, the splitting $\sigma$ induces a splitting $\mathrm{TM} \stackrel{\sigma}{\rightarrow} E / \mathrm{im} \partial$ which is a morphism of Lie algebroids. Ševera expressed the bracket of the Courant algebroid $E$ by means of the bracket of the Lie algebroid $E /$ im $\partial$.

The results obtained so far in this section straightforwardly extend to regular Courant algebroids. Indeed, such an algebroid $(E, g, \rho)$ is a transitive Courant algebroid over the base manifold $M$ of $E$ seen as the sum of the leaves of the foliation $\mathscr{F}=\operatorname{im} \rho$. Therefore, we get formulas (3.8) again. In order to ensure that we obtain brackets that are differentiable with respect to the original differentiable structure of $M$, it suffices to use metric connections and forms $\lambda, \Lambda$ that enjoy this kind of differentiability. Such connections are just Lie algebroid connections for the tangent Lie algebroid of $\mathscr{F}$ (called $\mathscr{F}$-partial connections or connections along the leaves of $\mathscr{F}$ in foliation theory). Thus, Propositions 3.2, 3.3, and Corollary 3.4, where we ask the anchor to be surjective over a regular foliation $\mathscr{F}$ of $M$ and the connection to be along the leaves of $\mathscr{F}$, describe all the regular (restricted) Courant algebroids.

\section{Miscellanies}

(a) The basic example of a skew-symmetric Courant algebroid appeared in [1]. It was the vector bundle $E=\mathrm{TM} \oplus T^{*} M$, endowed with the neutral pseudo-Euclidean metric

$$
g(X \oplus \alpha, Y \oplus \beta)=\frac{1}{2}(i(X) \beta+i(Y) \alpha),
$$

the nondegenerate cross-section $\omega \in \wedge^{2} E^{*}$,

$$
\omega(X \oplus \alpha, Y \oplus \beta)=\frac{1}{2}(i(X) \beta-i(Y) \alpha),
$$

the Courant bracket

$$
[X \oplus \alpha, Y \oplus \beta]=[X, Y] \oplus\left[L_{X} \beta-L_{Y} \alpha-d(\omega(X \oplus \alpha, Y \oplus \beta))\right],
$$


and the projection $\rho(X \oplus \alpha)=X$, where $X, Y \in \Gamma(\mathrm{TM}), \alpha, \beta \in \Gamma\left(T^{*} M\right)$, and, for clarity, we denoted an element $(X, \alpha)=X+\alpha \in \mathrm{TM} \oplus T^{*} M$ by $X \oplus \alpha$.

Straightforward computations show that the bracket (4.3), together with $g, \rho$, satisfies the conditions of Definition 1.6, and TM $\oplus T^{*} M$ is a skew-symmetric Courant algebroid.

For the same data, the formula

$$
(X \oplus \alpha) \star(Y \oplus \beta)=[X, Y] \oplus\left(L_{X} \beta-i(Y) d \alpha\right)
$$

defines a structure of a nonskew-symmetric Courant algebroid and the Courant bracket (4.3) is the skew-symmetric part of the bracket (4.4).

The Courant bracket was extended by Ševera and Weinstein [7] by the addition of a term of the form $i(X \wedge Y) \Phi$ to the $T^{*} M$-component of the right-hand side of (4.3), $\Phi$ being a closed 3-form on $M$.

The Courant algebroid structures described above are transitive, hence particular cases of the general formulas (3.7), (3.8). Indeed, in the present case, $\partial f=0 \oplus d f\left(f \in C^{\infty}(M)\right)$, $\operatorname{im} \partial=T^{*} M, C=\{0\}$, we may take $Q=\mathrm{TM}$, and a suitable connection is provided by any linear connection on $M$. Then, the first formula (3.7) becomes (4.3). Indeed, this is trivial for $\alpha=d f_{1}, \beta=d f_{2}\left(f_{1}, f_{2} \in C^{\infty}(M)\right)$, and it is true for any $\alpha, \beta$ because the two formulas (3.7) and (4.3) behave in the same way when arguments are multiplied by a function. The addition of a form $\lambda$ leads to the Ševera-Weinstein Courant bracket with $\Phi=-\left.2 \Lambda\right|_{Q}$. Since $C=\{0\}$, we necessarily are in the restricted case, $\lambda, \Lambda$ satisfy (1.22), and the conditions (3.14)-(3.16) reduce to $d \Phi=0$. Therefore, the Courant and ŠeveraWeinstein brackets define all the Courant algebroid structures on $\mathrm{TM} \oplus T^{*} M$ endowed with the metric (4.1) and the anchor $\rho(X \oplus \alpha)=X$.

However, $\mathrm{TM} \oplus T^{*} M$ may have more Courant algebroid structures if, for instance, we change the anchor, as we will see below.

In [4], the Courant bracket was extended to vector bundles $A \oplus A^{*}$, where $\left(A, A^{*}\right)$ is a Lie bialgebroid with anchors $\alpha, \alpha^{*}$, respectively, such that the extended bracket, the metric $g$ defined like in (4.1), and the anchor $\rho=\alpha+\alpha^{*}$ define a structure of skew-symmetric Courant algebroid. The extended bracket is

$$
\begin{aligned}
{\left[\left[a \oplus a^{*}, b \oplus b^{*}\right]\right]=} & \left\{[a, b]_{A}+L_{a^{*}}^{*} b-L_{b^{*}}^{*} a+d^{*}\left(\omega\left(a \oplus a^{*}, b \oplus b^{*}\right)\right)\right\} \\
& \oplus\left\{\left[a^{*}, b^{*}\right]_{A^{*}}+L_{a} b^{*}-L_{b} a^{*}-d\left(\omega\left(a \oplus a^{*}, b \oplus b^{*}\right)\right)\right\},
\end{aligned}
$$

where $d, L$, respectively, $d^{*}, L^{*}$, are the exterior differential and Lie derivative associated with the Lie algebroid structure of $A$, respectively, $A^{*}$, and $\omega$ is defined like in (4.2).

One can check that the bracket (4.5) is the skew-symmetric part of the product

$$
\begin{aligned}
& \left(a \oplus a^{*}\right) \star\left(b \oplus b^{*}\right) \\
& \quad=\left\{[a, b]_{A}+L_{a^{*}}^{*} b+i\left(b^{*}\right) d^{*} a\right\} \oplus\left\{\left[a^{*}, b^{*}\right]_{A^{*}}+L_{a} b^{*}-i(b) d a^{*}\right\} .
\end{aligned}
$$

It is possible to connect the bracket (4.5) with a metric connection as indicated in Proposition 2.1, but this does not seem to give interesting formulas. On the other hand, we may use the bracket (4.5) and Propositions 1.11 and 2.3 in order to derive new Courant algebroid structures on the vector bundles $A \oplus A^{*}$ and $A \oplus A^{*} \oplus C$ for any flat pseudoEuclidean bundle $C$. 
The Courant bracket (4.3) on $\mathrm{TM} \oplus \mathrm{TM}^{*}$ is the particular case of the bracket (4.5) where $A=$ TM with the Lie bracket and $A^{*}=T^{*} M$ with the zero bracket and zero anchor. But, if we assume that $P$ is a Poisson bivector field on $M$ and use the cotangent algebroid structure defined by $P$ on $T^{*} M$, we get a new Courant algebroid structure on TM $\oplus \mathrm{TM}^{*}$ with the same metric (4.1), with the anchor $I d_{\mathrm{TM}} \oplus \#_{P}$, and with the bracket (4.5). Again, we are in the transitive, restricted case, and it will be possible to express the bracket under the form (3.7). Indeed, the kernel of the anchor is

$$
K=\left\{X \oplus \alpha / X+\#_{P} \alpha=0\right\} \approx T^{*} M,
$$

and we may use the complementary subbundle $Q=\{X \oplus 0\} \approx$ TM such that a crosssection of $\mathrm{TM} \oplus \mathrm{TM}^{*}$ decomposes as follows:

$$
X \oplus \alpha=\left(\left(X+\sharp_{P} \alpha\right) \oplus 0\right)+\left(\left(-\sharp_{P} \alpha\right) \oplus \alpha\right) .
$$

It is easy to see that

$$
\partial f=\left(-X_{f}^{P}\right) \oplus d f, \quad f \in C^{\infty}(M),
$$

where $X_{f}^{P}$ is the Hamiltonian vector field of $f$ with respect to $P$. Then, by technical calculations, one checks that the brackets defined by the first formula (3.7) and by (4.5) coincide; the checks are to be made in each of the cases: two arguments in $Q$, one in $Q$ and one in im $\partial$, and two arguments in im $\partial$.

The original Courant bracket also leads to an example of a nonrestrictive, transitive pre-Courant algebroid. Assume that the manifold $M$ is endowed with a Riemannian metric $G$ and consider the vector bundle $E=\mathrm{TM} \oplus T^{*} M \oplus \mathrm{TM}$ with the anchor defined as the projection on the first term and the metric $g \oplus G$, where $g$ is given by (4.1). Then, $\partial f=0 \oplus d f \oplus 0$ and $E$ is a Courant vector bundle with surjective anchor, with the natural decomposition $E=(Q \oplus \operatorname{im} \partial) \oplus C$ where $Q=\mathrm{TM}$ and $C=(\mathrm{TM}, G)$, and the suitable connection defined by the Levi-Civita connection $\nabla$ of $G$, which satisfies $T_{\nabla, \rho}=0$.

Accordingly, we get a pre-Courant algebroid bracket on $\mathrm{TM} \oplus T^{*} M \oplus \mathrm{TM}$ if we use the corresponding formulas (3.7). The first formula (3.7) again yields the original Courant bracket and the remaining brackets (3.7) are determined by the Levi-Civita connection $\nabla$ of $G$ and the value of $\gamma$ as defined by (2.4), which yields

$$
\gamma\left(0 \oplus 0 \oplus Y_{1}, 0 \oplus 0 \oplus Y_{2}\right)=0 \oplus \xi\left(Y_{1}, Y_{2}\right) \oplus 0,
$$

where

$$
\xi\left(Y_{1}, Y_{2}\right)=G\left(Y_{1}, \nabla Y_{2}\right)-G\left(\nabla Y_{1}, Y_{2}\right)
$$

and $X_{a}, Y_{a} \in \Gamma$ TM, $\alpha_{a} \in \Gamma T^{*} M(a=1,2)$. Indeed, the $g \oplus G$-scalar product of the righthand side of (4.10) by any triple $X \oplus \alpha \oplus Y$ is the one prescribed by (2.4).

Now, let $\Phi$ be a differential 3-form on $M$ and define

$$
\begin{aligned}
\lambda\left(X_{1}\right. & \left.\oplus \alpha_{1} \oplus Y_{1}, X_{2} \oplus \alpha_{2} \oplus Y_{2}\right) \\
& =0 \oplus 2\left[i\left(X_{1} \wedge Y_{2}-X_{2} \wedge Y_{1}\right) \Phi\right] \oplus \sharp_{G}\left[i\left(X_{1} \wedge X_{2}\right) \Phi\right] .
\end{aligned}
$$


This form vanishes if one of the arguments belongs to im $\partial$ and

$$
\begin{aligned}
& \Lambda\left(X_{1} \oplus \alpha_{1} \oplus Y_{1}, X_{2} \oplus \alpha_{2} \oplus Y_{2}, X_{3} \oplus \alpha_{3} \oplus Y_{3}\right) \\
& \quad=\Phi\left(X_{1}, X_{2}, Y_{3}\right)+\Phi\left(X_{1}, Y_{2}, X_{3}\right)+\Phi\left(Y_{1}, X_{2}, X_{3}\right)
\end{aligned}
$$

is skew-symmetric. Therefore $\lambda$ of (4.12) may serve as an additional term that leads to a new pre-Courant algebroid bracket (3.8) on $\mathrm{TM} \oplus T^{*} M \oplus \mathrm{TM}$, which is not restricted. It will be a Courant algebroid bracket if and only if the conditions (3.9)-(3.12) hold. The significance of these conditions is unclear.

(b) The Courant bracket (4.3) may be defined for every Lie algebroid $A$ and it is the particular case of (4.5) where the dual bundle $A^{*}$ is endowed with the zero anchor and the zero bracket. In what follows, we use this remark in order to define a Courant algebroid structure on the tangent bundle of a para-Hermitian manifold $M$. We recall the definition [2]: a para-Hermitian structure on $M$ consists of a neutral metric $g$ on TM and a $(1,1)$ tensor field $F$ that satisfies the conditions

$$
\begin{gathered}
F^{2}=I=I d ., \quad g(F X, F Y)=-g(X, Y), \quad \forall X, Y \in \Gamma \mathrm{TM}, \\
N_{F}(X, Y)=[F X, F Y]-F[F X, Y]-F[X, F Y]+F^{2}[X, Y]=0 .
\end{gathered}
$$

Condition (4.15), which is the vanishing of the Nijenhuis tensor of $F$, is the integrability condition of the structure.

It follows that $M$ also has a nondegenerate 2-form

$$
\omega(X, Y)=g(F X, Y)
$$

which satisfies the condition

$$
\omega(F X, F Y)=-\omega(X, Y)
$$

and that $\mathrm{TM}=W_{+} \oplus W_{-}$, where the terms are the \pm 1 -eigendistributions of $F$ and are integrable because of (4.15). We will denote by

$$
F_{ \pm}=\frac{1}{2}(I \pm F)
$$

the projectors on $W_{ \pm}$, respectively. From (4.14) and (4.17), it follows that $W_{ \pm}$are maximal isotropic subbundles with respect to $g$ and Lagrangian subbundles with respect to $\omega$. Accordingly, the musical isomorphism $b_{g}$ sends $W_{ \pm}$onto the dual space $W_{\mp}^{*}$ and defines an isomorphism $\mathrm{TM} \approx W_{+} \oplus W_{+}^{*}$.

Because of integrability, $W_{+}$, with the Lie bracket, is a Lie algebroid and, if we use (4.3) in this case, we get a bracket on the tangent bundle TM defined by

$$
[X, Y]_{F_{+}}=\left[X_{+}, Y_{+}\right]+\#_{g}\left\{L_{X_{+}} b_{g} Y_{-}-L_{Y_{+}} b_{g} X_{-}-\frac{1}{2} d[\omega(X, Y)]\right\},
$$

where $X_{ \pm}=F_{ \pm} X, Y_{ \pm}=F_{ \pm} Y$. The conclusion is that $\left(\mathrm{TM}, g, F_{+},[\cdot, \cdot]_{F_{+}}\right)$is a regular, skewsymmetric Courant algebroid. 
Remark 4.1. In an obvious way, we may speak of para-Hermitian vector bundles and, for any differentiable manifold $M$, the bundle $\mathrm{TM} \oplus T^{*} M$ is para-Hermitian with the metric (4.1) and with $F$ defined by

$$
F(X \oplus \alpha)=X \oplus(-\alpha)
$$

Then, formulas (4.3) and (4.19) are similar.

(c) In what follows, we give some indications about the use of Proposition 2.1 for possible Courant algebroid structures on the tangent bundle of an arbitrary differentiable manifold $M$.

A Courant algebroid structure on TM requires a pseudo-Riemannian metric $g$ on $M$, and a field $\phi$ of endomorphisms of TM, which will be the anchor. Notice that $\partial f=$ $(1 / 2) \#_{g}(d f \circ \phi)$ and that $\operatorname{span}\{d f \circ \phi\}=\operatorname{ann} \operatorname{ker} \phi$. Hence, $\phi$ is a Courant anchor if and only if $\operatorname{im} \phi$ is a generalized, completely integrable distribution and $\#_{g}(\operatorname{ann} \operatorname{ker} \phi)$ is an isotropic distribution. In particular, we must have $\operatorname{rank} \phi \leq(1 / 2) \operatorname{dim} M$. Furthermore, one must have a bracket $[[X, Y]](X, Y,[[X, Y]] \in \Gamma T M)$ that satisfies properties (i), (iii), and (v) of Proposition 1.5. In Section 4(b), we had an example of such a situation.

Generally, on TM we have the Levi-Civita connection $\nabla$ of $g$, which is metric, and we may use it to express the bracket. The connection $\nabla$ has no torsion but it has a $\phi$-torsion

$$
T_{(\nabla, \phi)}(X, Y)=\phi\left(\nabla_{\phi X} Y-\nabla_{\phi Y} X\right)-[\phi X, \phi Y]=\left(\nabla_{\phi Y} \phi\right)(X)-\left(\nabla_{\phi X} \phi\right)(Y) .
$$

A technical calculation shows that the $\phi$-torsion is related with the Nijenhuis tensor $N_{\phi}$ (see (4.15)). Namely,

$$
T_{(\nabla, \phi)}(X, Y)=\left(\phi \circ \nabla_{Y} \phi\right)(X)-\left(\phi \circ \nabla_{X} \phi\right)(Y)-N_{\phi}(X, Y)
$$

The operator $\gamma$ of (2.4) will now be defined by

$$
g(\gamma(X, Y), Z)=\frac{1}{2}\left[g\left(X, \nabla_{\phi Z} Y\right)-g\left(\nabla_{\phi Z} X, Y\right)\right]
$$

and the Courant algebroid bracket will be

$$
[[X, Y]]=\nabla_{\phi X} Y-\nabla_{\phi Y} X-\gamma(X, Y)-\beta(X, Y)
$$

where $\beta$ is a tensor field of type $(1,2)$ on $M$ such that $\phi(\beta(X, Y))=T_{(\nabla, \phi)}(X, Y)$ and $B(X, Y, Z)=g(\beta(X, Y), Z)$ is a 3 -form on $M$.

Thus, essentially, a Courant algebroid structure on (TM, $g, \phi)$ is a special type of a 3form. What we must still ask is the fulfillment of condition $\mathscr{C}=0$ for $\mathscr{C}$ defined by (2.13). Unfortunately, this condition is too complicated and does not provide a practical way to find new Courant algebroids. This is true even if stronger conditions are added. For instance, if we ask $\phi$ to be $\nabla$-parallel along paths in the leaves of $\operatorname{im} \phi,(4.21)$ shows that 
$T_{(\nabla, \phi)}=0$, and we may consider the bracket (4.24) with $\beta=0$. As shown in Section 2 , the condition for this bracket to define a Courant algebroid structure is the annulation of the invariant $\mathscr{C}_{0}$ defined by formula (2.12), which, now, takes the form

$$
\sum_{\operatorname{Cycl}(X, Y, Z)}\left\{R_{\nabla}(\phi X, \phi Y) Z-\left(\nabla_{\phi Z} \gamma\right)(X, Y)-\gamma(\gamma(X, Y), Z)\right\}=0 .
$$

(d) Now, in a different direction, we refer to the case of what should be called foliated, regular Courant algebroids, and show another way of describing their brackets.

Let $M$ be a manifold endowed with a regular foliation $\mathscr{F}_{F}$. Let $E$ be a foliated, vector bundle over $M$ endowed with a foliated, pseudo-Euclidean metric $g$. This means that $E$ has a given, maximal system of local trivializations with transition functions that are constant along the leaves of $\mathscr{F}$ (foliated functions), and, for all $e_{1}, e_{2} \in \Gamma_{\mathrm{pr}} E$, where $\Gamma_{\mathrm{pr}}$ denotes the space of projectable cross-sections of $E$ (i.e., constant along the leaves), the function $g\left(e_{1}, e_{2}\right)$ is constant along the leaves (e.g., see [5] for the theory of foliations). Then, we have the following.

Proposition 4.2. On a foliated pair $(E, g)$, there exist metric connections $\nabla$ that satisfy the condition $\nabla_{X} e=0$, for all $X \in \Gamma T \mathscr{F}$ and for all $e \in \Gamma_{\mathrm{pr}} E$.

Proof. Take an arbitrary normal bundle $N \mathscr{F}$ (i.e., $\mathrm{TM}=T \mathscr{F} \oplus N \mathscr{F}$ ) and an arbitrary metric connection $\tilde{\nabla}$ of $(E, g)$. Then define

$$
\nabla_{X} e= \begin{cases}\tilde{\nabla}_{X} e & \forall X \in \Gamma N \mathscr{F}, \forall e \in \Gamma E \\ 0 & \forall X \in \Gamma T \mathscr{F}, \forall e \in \Gamma_{\mathrm{pr}} E\end{cases}
$$

It is easy to check that this produces a connection as required. Indeed, we must still define $\nabla_{X} e$ for $X \in \Gamma T \mathscr{F}$ and a nonprojectable $e \in \Gamma E$. For this purpose, we take local projectable bases $e_{\alpha}$ of $E$ and, for

$$
e=\sum_{\alpha} f_{\alpha} e_{\alpha}, \quad e_{\alpha} \in \Gamma_{\mathrm{pr}} E, f_{\alpha} \in C^{\infty}(M)
$$

we put

$$
\nabla_{X} e=\sum_{\alpha}\left(X f_{\alpha}\right) e_{\alpha}
$$

A connection that satisfies the properties stated by Proposition 4.2 will be called an adapted connection of $(E, g)$.

Let $(E, g)$ be foliated and we assume that there exists a surjective morphism $\rho: E \rightarrow T \mathscr{F}$, which is a Courant anchor of $(E, g)$. Then, for any adapted connection $\nabla$, one has a nonzero $\rho$-torsion given by

$$
T_{(\nabla, \rho)}\left(e_{1}, e_{2}\right)=-\left[\rho e_{1}, \rho e_{2}\right], \quad e_{1}, e_{2} \in \Gamma_{\mathrm{pr}} E .
$$


Formula (2.4) yields the operator

$$
\gamma\left(e_{1}, e_{2}\right)=0, \quad e_{1}, e_{2} \in \Gamma_{\mathrm{pr}} E,
$$

and (2.3) gives a bracket such that

$$
\left[e_{1}, e_{2}\right]_{0}=0, \quad e_{1}, e_{2} \in \Gamma_{\mathrm{pr}} E
$$

The values of this bracket for arbitrary cross-sections $f_{1} e_{1}, f_{2} e_{2}, e_{1}, e_{2} \in \Gamma_{\mathrm{pr}} E, f_{1}, f_{2} \in$ $C^{\infty}(M)$ follow from property (iv), Proposition 1.5. Furthermore, the operator $\gamma$ satisfies the condition $\mathscr{C}_{0}=0$, where $\mathscr{C}_{0}$ is defined by (2.12); this is obvious for projectable arguments and is true for arbitrary arguments because $\mathscr{C}_{0}$ is a tensor. Hence, we get the following.

Proposition 4.3. For a triple $(E, g, \rho)$ as described above, a Courant algebroid bracket is a bracket of the form (2.5), where $\beta \in \Gamma\left(\wedge^{2} E^{*} \otimes E\right)$ is associated with a 3-form $B \in \Gamma\left(\wedge^{3} E^{*}\right)$ and satisfies the conditions

$$
\begin{aligned}
\rho\left(\beta\left(e_{1}, e_{2}\right)\right) & =-\left[\rho e_{1}, \rho e_{2}\right], \\
\sum_{\operatorname{Cycl}(1,2,3)} \beta\left(\beta\left(e_{1}, e_{2}\right), e_{3}\right) & =\sum_{\operatorname{Cycl}(1,2,3)}\left[\beta\left(e_{1}, e_{2}\right), e_{3}\right]_{0}
\end{aligned}
$$

for all $e_{1}, e_{2}, e_{3} \in \Gamma_{\mathrm{pr}} E$.

Condition (4.33) exactly is the annulation of the invariant $\mathscr{C}$ defined by formula (2.13), where we took into consideration (4.31) and the fact that $\mathscr{C}_{0}=0$. It was shown in Section 2 that $\mathscr{C}=0$ characterizes Courant algebroids.

\section{Appendix}

\section{Dirac linear spaces}

The Courant algebroids resulted from the process of studying Dirac structures, which are a significant generalization of the Poisson structures [1]. Although this is not a subject of the present paper, we have added this appendix, which shows that the known linear algebra of Dirac structures is a part of para-Hermitian linear algebra.

A para-Hermitian vector space is a $2 n$-dimensional vector space $W$ that has the structure indicated for the fibers of the tangent bundle of a para-Hermitian manifold in the previous section. On $W$ we have the ingredients $g, \omega, F, W_{ \pm}, F_{ \pm}$, with the algebraic properties stated in Section $4(\mathrm{~b})$, and $W=W_{+} \oplus W_{-}, b_{g}: W_{ \pm} \approx W_{+}^{*}$.

The space $W$ has adapted bases $\left(b_{i}, c_{j}\right)(i, j=1, \ldots, n)$, where $\left(b_{1}, \ldots, b_{n}\right)$ is a basis of $W_{+}$, therefore, $b_{g} b_{1}, \ldots, b_{g} b_{n}$ is a basis of $W_{-}^{*}$, and $\left(c_{1}, \ldots, c_{n}\right)$ is the corresponding dual basis of $W_{-}$, that is, $g\left(b_{i}, c_{j}\right)=\delta_{i j}(i, j=1, \ldots, n)$.

Definition A.4. (1) [1] A maximal, $g$-isotropic subspace $L$ of the para-Hermitian vector space $W$ is called a Dirac subspace of $W$. (2) [3] A pair $\left(L, L^{\prime}\right)$ of complementary Dirac subspaces of $W\left(W=L \oplus L^{\prime}\right)$ is a reflector in $W$. 
Proposition A.5. For any Dirac subspace $L \subseteq W$, there exists a family $\mathscr{T}(L)$ of Dirac subspaces that are complementary to $L$ in $W$, and $\mathscr{T}(L)$ is an affine space modelled over the linear space of the skew-symmetric matrices of order $n$.

Proof. The results are analogous to known results for Lagrangian subspaces of a symplectic vector space, and we prove them as in the latter case, for example, [9]. For any subspace $S \subseteq W$ such that $W=L \oplus S$ and any basis $\left(l_{1}, \ldots, l_{n}\right)$ of $L$, there exists a unique conjugated basis $\left(s_{1}, \ldots, s_{n}\right)$ of $S$, such that $g\left(l_{i}, s_{j}\right)=\delta_{i j}$. Using the conjugated basis, we can obtain vectors

$$
u_{i}=s_{i}+\tau_{i}^{k} l_{k}
$$

(the Einstein summation convention holds) such that $g\left(u_{i}, u_{j}\right)=0$, and these vectors span a Dirac subspace $L^{\prime}$ that satisfies $L \oplus L^{\prime}=W$. Furthermore, if $L^{\prime \prime}$ is another Dirac subspace such that $L \oplus L^{\prime \prime}=W$ and if $\left(u_{i}\right)$ is the conjugated basis of $\left(l_{i}\right)$ in $L^{\prime}$ and $\left(v_{i}\right)$ is the conjugated basis of $\left(l_{i}\right)$ in $L^{\prime \prime}$, there exists a unique skew-symmetric matrix $\left(\theta_{i}^{j}\right)$ such that $v_{i}=u_{i}+\theta_{i}^{j} l_{j}$.

Using adapted bases of $W$, it follows that the set of the reflectors of $W$ is the $n(n-1)$ dimensional homogeneous space $\mathscr{R}=O(W, g) / \mathrm{pH}(W) \approx O(n, n) / \mathrm{Gl}(n)$, where $O(W, g)$ is the $g$-preserving subgroup of the general linear group $\mathrm{Gl}(W)$, which acts transitively on $\mathscr{R}, \mathrm{pH}(W)$ is the para-Hermitian subgroup, which commutes with $F$ and is the isotropy subgroup of the pair $\left(W_{+}, W-\right) \in \mathscr{R}, O(n, n) \approx O(W, g)$ is the subgroup of $\operatorname{Gl}(2 n)$ which preserves the canonical neutral metric, and $\mathrm{Gl}(n) \approx \mathrm{pH}(W)$ by the embedding

$$
A \longmapsto\left(\begin{array}{cc}
A & 0 \\
0 & { }^{t} A^{-1}
\end{array}\right) \quad(A \in \mathrm{Gl}(n))
$$

see $[3,11]$.

Proposition A.5 shows that the set $\mathscr{D}$ of Dirac subspaces of $W$ is the quotient space of $\mathscr{R}$ by the equivalence relation with equivalence classes $\mathscr{T}(L)$, hence $\mathscr{D}$ is a $[n(n-$ $1) /] 2$-dimensional space, namely, the homogeneous space $O(W, g) / O_{W_{+}}(W, g)$, where the isotropy group of $W_{+} \in \mathscr{D}$ at the denominator is that of the elements $\phi \in O(W, g)$ which satisfy the condition $F_{-} \circ \phi \circ F_{+}=0$.

We also notice that $L \subseteq W$ is a Dirac subspace if and only if $F(L)$ is the $\omega$-orthogonal subspace of $L$. Therefore, if $L$ is a Dirac subspace, $\operatorname{ker}\left(\left.\omega\right|_{L}\right)=L \cap F(L)$. On the other hand, it follows easily that for a Dirac subspace $L$, one has

$$
\operatorname{ker}\left(\left.\omega\right|_{L}\right)=\left(W_{+} \cap L\right) \oplus\left(W_{-} \cap L\right)
$$

The following proposition shows that the integers $k=\operatorname{dim}\left(W_{-} \cap L\right)$ and $r=\operatorname{rank}\left(\left.\omega\right|_{L}\right)$ are the only invariants of a Dirac subspace with respect to the action of the paraHermitian subgroup $\mathrm{pH}(W)$. 
Proposition A.6. The group $\mathrm{pH}(W)$ acts transitively on the set of Dirac subspaces $L$ with given values $k, r$.

Proof. Denote $p_{ \pm}=\left.F_{ \pm}\right|_{L}$. Obviously, $\operatorname{ker} p_{ \pm}=W_{\mp} \cap L$, and we get two linear spaces

$$
L_{ \pm}=\operatorname{im} p_{ \pm} \approx L / W_{\mp} \cap L
$$

of dimension $n-\operatorname{dim}\left(W_{\mp} \cap L\right)$. Since $W_{\mp} \cap L \subseteq \operatorname{ker}\left(\left.\omega\right|_{L}\right)$, we see that the subspaces $L_{ \pm}$ have induced, skew-symmetric, bilinear forms $\omega_{ \pm}^{L}$.

Moreover, because of the structure of $\operatorname{ker}\left(\left.\omega\right|_{L}\right)$ as described above, we see that rank $\omega_{ \pm}^{L}=\left.\operatorname{rank} \omega\right|_{L}$ and $\operatorname{ker} \omega_{ \pm}^{L}=p_{ \pm}\left(W_{ \pm} \cap L\right)$. (Clearly, $\left.p_{ \pm}\right|_{W_{ \pm} \cap L}$ have kernel zero and are isomorphisms onto the corresponding images.)

As shown in [1], it is possible to reconstruct $L$ from each of the pairs $\left(L_{ \pm}, \omega_{ \pm}^{L}\right)$, namely,

$$
\begin{aligned}
& L=\left\{w \in W / F_{+}(w) \in L_{+}, g\left(F_{-}(w), u\right)=-\frac{1}{2} \omega_{+}^{L}\left(F_{+}(w), u\right), \forall u \in L_{+}\right\}, \\
& L=\left\{w \in W / F_{-}(w) \in L_{-}, g\left(F_{+}(w), u\right)=\frac{1}{2} \omega_{-}^{L}\left(F_{-}(w), u\right), \forall u \in L_{-}\right\} .
\end{aligned}
$$

The two formulas have similar justifications, and we refer to the first only. A straightforward check shows that, for any choice of a pair $\left(L_{+}, \omega_{+}\right)$, which consists of an arbitrary subspace of $W$ and an arbitrary 2 -form on that subspace, $L$ defined by the first formula is an isotropic subspace of $W$, that $F_{+}(L)=L_{+}$, and that $\omega_{+}$is induced by $\omega$. Furthermore, the formula implies

$$
\left.\operatorname{ker} F_{+}\right|_{L}=L \cap W_{-}=\left\{w \in W_{-} / g(w, u)=0, \forall u \in L_{+}\right\},
$$

and, since $\left.g\right|_{W_{-} \times W_{+}}$is a nondegenerate pairing, $k=n-\operatorname{dim}\left(L_{+}\right)$. Accordingly, $\operatorname{dim} L=$ $\operatorname{dim}\left(\left.\operatorname{ker} F_{+}\right|_{L}\right)+\operatorname{dim}\left(\left.\operatorname{im} F_{+}\right|_{L}\right)=n$ and $L$ is the required Dirac subspace.

Now, if $L, L^{\prime}$ are Dirac subspaces of $W$ with the same invariants $k, r$, there exists a transformation $\psi \in \mathrm{Gl}\left(W_{+}\right)$which sends the pair $\left(L_{+}, \omega_{+}^{L}\right)$ onto $\left(L_{+}^{\prime}, \omega_{+}^{L^{\prime}}\right)$. Obviously, the image of $\psi$ in $\mathrm{pH}(W)$ via the embedding (A.2) sends $L$ onto $L^{\prime}$.

Of course, instead of the invariant $k=\operatorname{dim}\left(W_{-} \cap L\right)$, we may consider $h=\operatorname{dim}\left(W_{+} \cap\right.$ $L)$. These two numbers are related by $k+h=n-r$.

\section{References}

[1] T. J. Courant, Dirac manifolds, Trans. Amer. Math. Soc. 319 (1990), no. 2, 631-661.

[2] V. Cruceanu, P. Fortuny, and P. M. Gadea, A survey on paracomplex geometry, Rocky Mountain J. Math. 26 (1996), no. 1, 83-115.

[3] G. R. Jensen and M. Rigoli, Neutral surfaces in neutral four-spaces, Matematiche (Catania) 45 (1990), no. 2, 407-443 (1991).

[4] Z.-J. Liu, A. Weinstein, and P. Xu, Manin triples for Lie bialgebroids, J. Differential Geom. 45 (1997), no. 3, 547-574.

[5] P. Molino, Riemannian Foliations, Progress in Mathematics, vol. 73, Birkhäuser Boston, Massachusetts, 1988.

[6] D. Roytenberg, Courant algebroids, derived brackets and even symplectic supermanifolds, Ph.D. thesis, University of California, Berkeley, 1999, http://www.arXiv.org/abs/ math.DG/9910078. 


\section{Transitive Courant algebroids}

[7] P. Ševera and A. Weinstein, Poisson geometry with a 3-form background, Progr. Theoret. Phys. Suppl. (2001), no. 144, 145-154.

[8] K. Uchino, Remarks on the definition of a Courant algebroid, Lett. Math. Phys. 60 (2002), no. 2, 171-175.

[9] I. Vaisman, Symplectic Geometry and Secondary Characteristic Classes, Progress in Mathematics, vol. 72, Birkhäuser Boston, Massachusetts, 1987.

[10] Lectures on the Geometry of Poisson Manifolds, Progress in Mathematics, vol. 118, Birkhäuser, Basel, 1994.

[11] Variations on the theme of twistor spaces, Balkan J. Geom. Appl. 3 (1998), no. 2, 135156.

Izu Vaisman: Department of Mathematics, University of Haifa, 31905 Haifa, Israel

E-mail address: vaisman@math.haifa.ac.il 


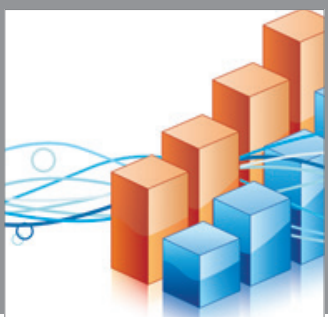

Advances in

Operations Research

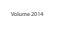

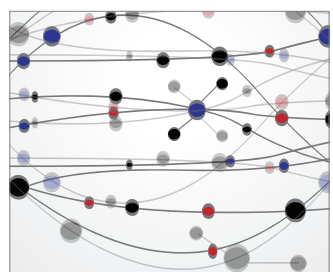

\section{The Scientific} World Journal
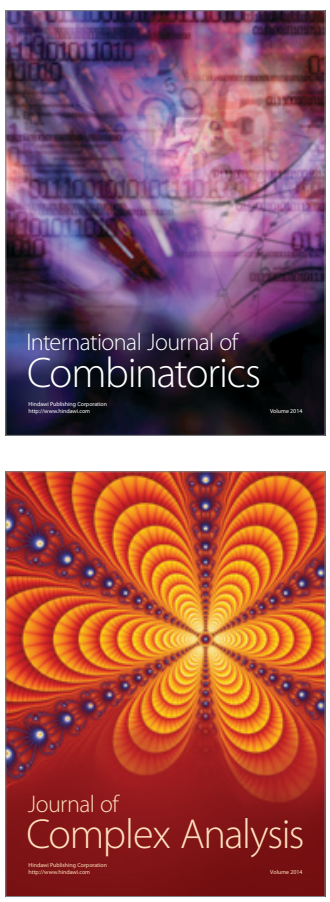

International Journal of

Mathematics and

Mathematical

Sciences
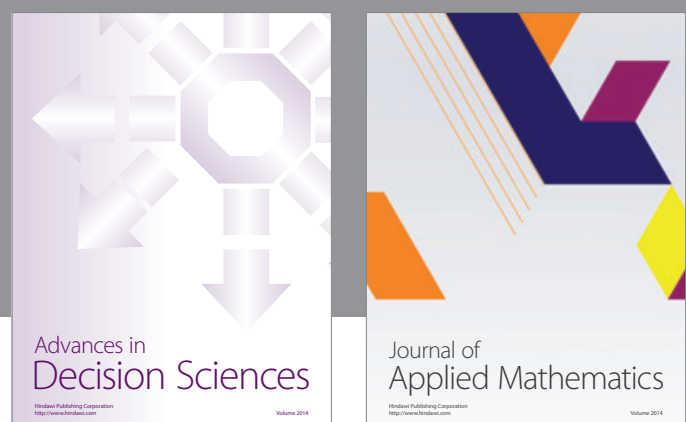

Journal of

Applied Mathematics
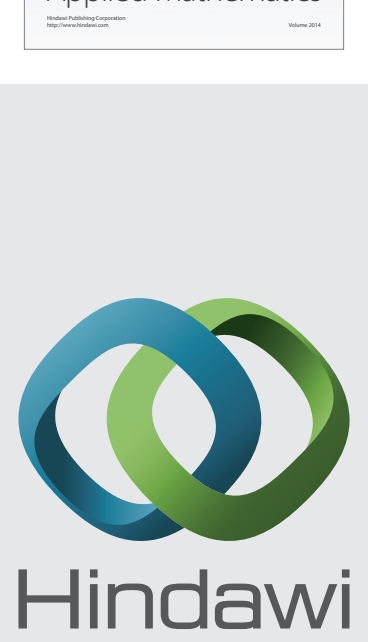

Submit your manuscripts at http://www.hindawi.com
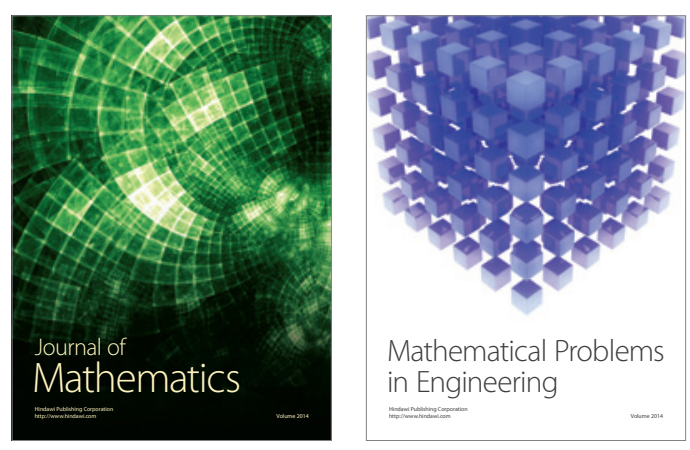

Mathematical Problems in Engineering
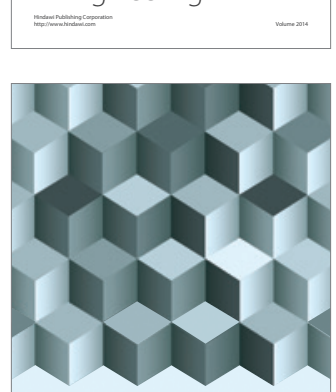

Journal of

Function Spaces
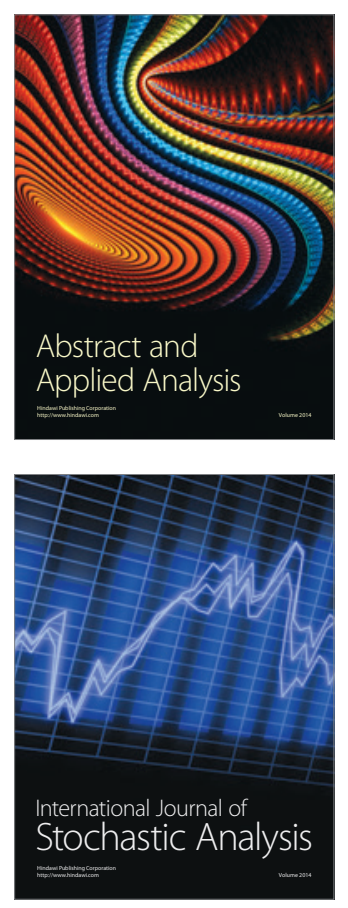

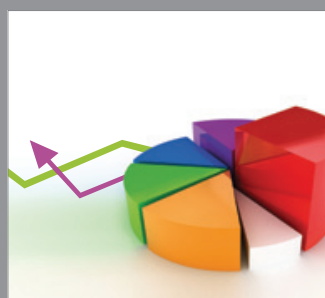

ournal of

Probability and Statistics

Promensencen
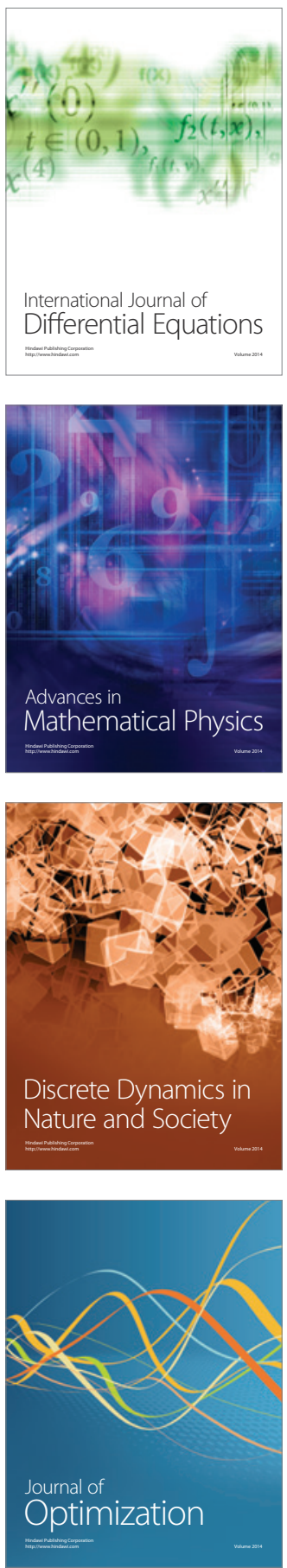